\title{
Fraktion der CDU/CSU im Deutschen Bundestag
}

\author{
Dorothee Bär, MdB \\ Sprecherin der CDU/CSU-Bundestagsfraktion für Familie, Senioren, Frauen und \\ Jugend $\mid$ dorothee.baer@bundestag.de |www.dorothee-baer.de $\mid$ www.cducsu.de
}

Nach dem Aussetzen des Zivildienstes ist es uns gelungen, mit dem neuen Bundesfreiwilligen- Fraktion im Deutschen Bundestag dienst ein überaus erfolgreiches neues Instrument freiwilligen Engagements zu etablieren. Der Zuspruch hat alle Erwartungen übertroffen. Es hat sich gezeigt, dass sich junge Menschen keineswegs nur dann engagieren, wenn dieses Engagement als Ersatz für den Wehrdienst quasi verpflichtend war, sondern dass sie dies aus freien Stücken und mit Leidenschaft tun.

Die bewährten Jungendfreiwilligendienste haben unter dieser Entwicklung nicht gelitten - ganz im Gegenteil. Dadurch, dass wir für den Bundesfreiwilligendienst die erprobten Strukturen der Jugendfreiwilligendienste sinnvoll erweitert haben, haben wir freiwilliges Engagement in seiner Vielfalt gestärkt - im neuen Dienst können sich auch ältere Menschen engagieren. Auch die Möglichkeit, einen Freiwilligendienst im Ausland zu leisten entweder im neuen Internationalen Jugendfreiwilligendienst, im entwicklungspolitischen Freiwilligendienst weltwärts oder auch in den Jugendfreiwilligendiensten im Ausland - trägt zur Vielfalt bei.

Freiwillige werden bei ihrem Einsatz von Fachkräften betreut und unterstützt. In begleitenden Seminaren - gesetzlich vorgeschrieben sind bei einem zwölfmonatigen Freiwilligendienst 25 Seminartage - lernen sie andere Freiwillige kennen und können Erfahrungen austauschen. Parallel zum Bundesfreiwilligendienst wurden auch die Jugendfreiwilligendienste als Bildungs- und Orientierungsangebote ausgebaut und finanziell gestärkt.

Diese neuen Entwicklungen in den Freiwilligendiensten rufen nach angemessener Reflexion. Eine Zeitschrift wie die Herausgeber von Voluntaris sie konzipiert haben, die einen Austausch zwischen akademischen und anwendungsbezogenen Perspektiven ermöglichen soll, kann dazu einen Beitrag leisten. Hier können Träger, Einsatzstellen, Verbände, pädagogische Fachkräfte, Politikerinnen und Politiker und natürlich die Freiwilligen selbst zu Wort kommen. Wichtig für mich ist dabei, dass das große Engagement der 
Freiwilligen durch zu akademische und verkopfte Debatten nicht kleingeredet und vor allem nicht zerredet wird. Dies wäre die einzige Sorge, die mir ein betont wissenschaftlicher Ansatz bereiten könnte: Bloß keine Überhöhung freiwilligen Engagements durch theorielastige Verwissenschaftlichung, sondern lieber ganz praktische Fragen in den Vordergrund rücken! Gespräche über die Organisation des freiwilligen Engagements sollen geführt, die Rolle des Staates in den Freiwilligendiensten angesprochen werden, aber eigentlich ist es doch ganz einfach: Von Freiwilligendiensten profitieren alle! Wer sich engagiert, tut nicht nur anderen etwas Gutes, sondern auch sich selbst. Denn diejenigen, die sich freiwillig für eine gute Sache engagieren, lernen viel für ihr privates und berufliches Leben oder ziehen Zufriedenheit daraus, dass sie andere Menschen an ihrer Lebenserfahrung teilhaben lassen. Die Menschen, die von Freiwilligen betreut werden, erfahren zusätzliche Aufmerksamkeit und Zuwendung und die Gesellschaft profitiert als Ganzes, wenn sich Menschen für andere Menschen oder für bestimmte Projekte einsetzen.

\title{
Engagement Global gGmbH - Service für Entwicklungsinitiativen
}

\author{
Gabriela Büssemaker \\ Hauptgeschäftsführerin der Engagement Global gGmbH \\ gabriela.buessemaker@engagement-global.de|www.engagement-global.de
}

Jeder der gehört werden will, muss reden. Jeder Redner braucht Zuhörer. Das ist das einfache Prinzip jedes Gesprächs. Damit

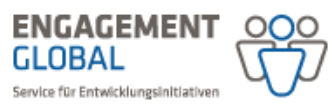
Menschen überhaupt miteinander ins Gespräch kommen, braucht es Gemeinsamkeiten - ob Interessen, Ideen oder Erfahrungen.

Freiwilligendienste bringen Menschen zusammen, die etwas in der Gesellschaft verändern und Verantwortung übernehmen wollen. Die Berichte der Freiwilligen über ihre Erlebnisse und Eindrücke sind es, die andere zuhören lassen. Sie geben neue Denkanstöße für die Bewältigung gesellschaftspolitischer Herausforderungen. Freiwilligendienste stehen dabei selbst vor neuen 